\title{
Mortalidade por aborto no Estado do Paraná: 1998 a 2004
}

\section{Mortality caused by abortion in the State of Paraná between 1998 and 2004}

\author{
Mortalidad causada por aborto en el Estado de Paraná entre 1998 y 2004
}

\author{
Jéssica Carvalho de Matos', Geisa dos Santos Luz", Sandra Marisa Pelloso"', Maria Dalva de Barros \\ Carvalho'v
}

\section{RESUMO}

As complicações do aborto destacam-se como uma das principais causas de mortalidade materna e apresenta um maior índice de subinformação. Este estudo objetivou analisar a evolução dos óbitos de mulheres que morreram por complicações do aborto no Estado do Paraná e no município de Maringá/PR, no período de 1998 a 2004. Tratase de um estudo descritivo, retrospectivo realizado por meio do levantamento dos óbitos maternos por causas relacionadas ao aborto no Estado do Paraná (PR) e município de Maringá/PR. Os óbitos por aborto no Estado do Paraná totalizaram no período um percentual de $6,27 \%$ dos óbitos maternos ocorridos, com destaque para a faixa etária de 30 a 39 anos. O estado civil foi bem distribuído entre solteiras e casadas. A raça branca predominou com $76,47 \%$. No município de Maringá, dentre o total de óbitos maternos identificou-se somente dois óbitos por aborto: mulheres solteiras com mesmo nível de escolaridade, porém com faixa etária distinta (18 e 42 anos). A evolução das mortes maternas por complicações do aborto no período estudado, aponta para a necessidade de uma ação urgente de caráter preventivo nos programas de saúde da mulher.

Palavras chave: Aborto; Mortalidade materna; Saúde da mulher.

\section{ABSTRACT}

The complications caused by abortion demonstrate they are among the main causes of women's death and they also present a higher level of deficient access to informationThis paper analyzes the evolution of the women's deaths caused by abortion in the State of Paraná and in Maringá in the period between 1998 and 2004. This descriptive and retrospective study was accomplished with the collection of data from the registration of the maternal deaths with causes related to abortion in the State of Paraná and in Maringá. The deaths caused by abortion in Paraná in that period corresponded to $6,27 \%$ of the total maternal deaths and they occurred specially amongst women at the age group from 30 to 39 years old. The marital status was well distributed among single and married women. The white race prevailed with $76,47 \%$. In Maringá, from the total of maternal deaths registered, two deaths were caused by abortion: single women with same school level, however in distinct age groups (18 and 42 years old). The evolution of the women's deaths caused by abortion in the period studied shows the need of an urgent preventive action within the women's health programs.

Key words: Abortion; Maternal mortality; Woman's health.

\section{RESUMEN}

Las complicaciones del aborto demuestran estar entre las causas principales de mortalidad maternal y también presentan un nivel más alto del acceso deficiente a información. Este estudio analiza la evolución de las muertes de mujeres causadas por aborto en el Estado de Paraná y específicamente en la ciudad de Maringá en el período entre 1998 y 2004 . Este estudio descriptivo y retrospectivo fue realizado con la colecta de datos de la matrícula de las muertes maternales con causas relacionadas al aborto en el Estado de Paraná y en Maringá. Las muertes causadas por aborto en Paraná en ese período correspondieron a $6.27 \%$ del total de las

\footnotetext{
'Enfermeira, Professora do Departamento de Enfermagem da Faculdade Intermunicipal do Noroeste do Paraná. Email: jecarvalhomatos@yahoo.com.br.

"Enfermeira, Professora do Departamento de Enfermagem da Faculdade Intermunicipal do Noroeste do Paraná. Email: geisaluz@yahoo.com.br

III Enfermeira, Doutora, Professora do Departamento de Enfermagem da Universidade Estadual de Maringá. E-mail: smpelloso@uem.br

Iv Enfermeira, Doutora, Professora do Departamento de Enfermagem da Universidade Estadual de Maringá. E-mail: mdbcarvalho@uem.br
} 
Matos JC, Luz GS, Pelloso SM, Carvalho MDB. Mortalidade por aborto no Estado do Paraná: 1998 a 2004 . Revista Eletrônica de Enfermagem [serial on line] 2007 Set-Dez; 9(3):806-814. Available from: URL: http://www. fen.ufg. br/revista/v9/n3/v9n3a19. htm

muertes maternales y ocurrieron especialmente entre mujeres del grupo de edad de 30 a 39 años. El estado civil se distribuyó bien entre mujeres solteras y casadas. La piel blanca prevaleció con $76.47 \%$. En Maringá, del total de muertes maternales registradas, dos fueron causadas por el aborto: mujeres solteras con mismo nivel de estudios, sin embargo de grupos de edad distintos (18 y

\section{NTRODUÇÃO}

O impacto da assistência à saúde da mulher é detectado pelos indicadores da saúde feminina, dentre eles, a Mortalidade Materna. A mortalidade materna está relacionada com a má qualidade da assistência no pré-natal, no parto e puerpério ${ }^{(1)}$, assim como ao coeficiente de mortalidade infantil e alta incidência de desnutrição, uma vez que a ausência da figura materna restringe o acesso ao aleitamento e ao carinho da mãe ${ }^{(2)}$.

A Morte Materna é conceituada pela Organização Mundial de Saúde como "a morte de uma mulher durante a gestação ou dentro de um período de 42 dias após o término da gestação, independentemente de duração ou da localização da gravidez, devida a qualquer causa relacionada com ou agravada pela gravidez ou por medidas tomadas em relação a ela, porém não devidas a causas acidentais ou incidentais". A partir da 10ạ Revisão do Código Internacional de Doenças (CID) foi instituída a classificação de Morte Materna tardia que ocorre entre 42 dias e 365 dias pós-parto ${ }^{(3)}$.

A Mortalidade Materna é classificada como: morte materna obstétrica (direta e indireta) e não obstétrica. Morte Materna obstétrica direta é aquela resultante de complicações obstétricas na gravidez. A Morte Materna obstétrica indireta está relacionada a doenças pré-existentes agravadas pelo estado
42 años). La evolución de las muertes de madres causadas por aborto en el periodo estudado apuntan la necesidad de una acción preventiva urgente en los programas de salud de la mujer.

Palabras clave: Aborto; Mortalidad maternal; Salud de la mujer.

fisiológico da gravidez. Quanto a Morte Materna não obstétrica esta pode ocorrer no momento da gravidez, parto ou puerpério por causas incidentais ou acidentais (suicídios, atropelamento, homicídio, dentre outros) ${ }^{(3)}$.

O coeficiente de Mortalidade Materna aceitável pelos órgãos de saúde no mundo está cerca de 10 Mortes Maternas/100000 Nascidos Vivos (NV) ${ }^{(4)}$.

No Brasil, o coeficiente de morte materna corresponde a $64,84 / 100000$ NV o que revela uma realidade preocupante. Entretanto, com a instituição dos Comitês de Mortalidade Materna, que fiscalizam continuamente os casos, este cenário tende a mudar ${ }^{(5)}$.

A taxa de Mortalidade Materna é influenciada sobremaneira pela situação sócioeconômica, portanto avalia também as condições de vida da mulher nas comunidades. Pesquisa realizada no município de São Paulo diagnosticou esta tendência. Um estudo aplicado em cinco regiões revelou que as mais carentes tinham o dobro de óbitos do que as regiões com melhores condições sócioeconômicas ${ }^{(6)}$.

Outro fator importante neste processo é a deficiência no incremento dos dados no Sistema de Informação de Mortalidade Materna pelos serviços de saúde, ou seja, a subnotificação dos casos. Em outro estudo sobre mortalidade materna no município de 
Matos JC, Luz GS, Pelloso SM, Carvalho MDB. Mortalidade por aborto no Estado do Paraná: 1998 a 2004 . Revista Eletrônica de Enfermagem [serial on line] 2007 Set-Dez; 9(3):806-814. Available from: URL: http://www. fen.ufg. br/revista/v9/n3/v9n3a19. htm

São Paulo durante o ano de 1996, verificou-se que mesmo existindo Comitês de Mortalidade Materna atuantes, a subnotificação do óbito materno estava próxima de $50 \%$ a $60 \%{ }^{(7)}$. Estima-se que há freqüência de $15 \%$ de subnotificação dos óbitos maternos na população brasileira. Vale justificar que as regiões que colaboram para este alto índice, pela precariedade de registros de todos os tipos de óbitos, são o Norte e Nordeste ${ }^{(8)}$. Esta situação pode ser justificada pelo despreparo dos profissionais médicos no preenchimento nas declarações de óbito, existência de cemitérios clandestinos e ausência de registros de nascidos vivos ${ }^{(1)}$.

Dentre todas as causas de óbito materno, as complicações do aborto apresentam uma maior subinformação ${ }^{(9)}$. Este déficit pode estar relacionado aos aspectos culturais, religiosos, legais e morais que inibem a população feminina em assumir o abortamento.

Dados oficiais relatamque no Brasil o aborto ocorre em $31 \%$ das gestações, ocorrência anual de 1.443 .350 e taxa de 3,7 abortos/100 mulheres de 15 a 49 anos. Comparado a países da Europa Ocidental, onde o aborto é realizado de maneira legal e acessível, estes números revelam-se desastrosos na vivência destas mulheres ${ }^{(10)}$. Assevera-se a estimativa de 1,4 milhões de abortos clandestinos por ano no Brasil, o que contribui para os altos índices de Mortalidade Materna (11).

Como reflexo dessa situação, no ano de 2004, 243.998 internações na rede SUS foram motivadas por curetagens pós-aborto, correspondentes aos casos de complicações decorrentes de abortamentos espontâneos e inseguros ${ }^{(10)}$.

A mulher ao decidir abortar leva em conta várias circunstâncias, tais como: estabilidade afetiva, condições financeira e social, perspectiva de vida, pressão familiar, dentre outras (11). Estes fatores são mais importantes na decisão pelo abortamento do que a possibilidade de complicações deste procedimento incluindo a morte. Este contexto pode explicar o alto índice de óbito materno por aborto, muitas das vezes, ilegal segundo o Código Penal Brasileiro.

O aborto no Brasil é uma prática considerada ilegal. O art. 128 do Código Penal determina que existe somente duas situações em que o aborto é considerado legal: se for a única forma de salvar a vida da mulher grávida ou se a gravidez foi resultado de um estupro ou incesto. Além disso, em alguns casos de má-formação congênita é previsto autorização do abortamento, porém raramente ocorre devido à morosidade do processo jurídico, geralmente autorizado após o nascimento da criança ${ }^{(12)}$.

Esta condição limita as opções de um aborto seguro e adequado na rede pública de saúde a qual está submetida a maior parte da população feminina, contribuindo sensivelmente para o aumento de óbitos maternos por complicações do aborto entre essas mulheres.

Diante das particularidades do abortamento no Brasil, este estudo visa analisar a evolução dos óbitos por causas relacionadas ao aborto no Estado do Paraná e no município de Maringá/PR, no período de 1998 a 2004. 
Matos JC, Luz GS, Pelloso SM, Carvalho MDB. Mortalidade por aborto no Estado do Paraná: 1998 a 2004 . Revista Eletrônica de Enfermagem [serial on line] 2007 Set-Dez; 9(3):806-814. Available from: URL: http://www. fen.ufg. br/revista/v9/n3/v9n3a19.htm

\section{MÉTODOS}

Trata-se de um estudo descritivo, retrospectivo dos óbitos maternos por causas relacionadas ao aborto no Estado do Paraná (PR) e município de Maringá/PR.

Foram levantadas todas as mortes maternas de mulheres de 10 a 49 anos ocorridas no período de 1998 a 2004, identificando todas as que ocorreram por complicações do aborto. Considerou-se todos os tipos de aborto (retido,espontâneo, induzido com indicação legalmente admitida (CID 10).

A coleta dos dados do estado do Paraná foi realizada no Sistema de Mortalidade Materna, Ministério da Saúde (SIM/MS/DATASUS), por meio do programa TABX fornecido pelo DATASUS. O SIM é um importante instrumento de monitoramento dos óbitos que permite identificar as principais causas de morte registradas nos municípios, estados e regiões brasileiras. Os dados de Maringá foram fornecidos pela 15aㅡ Regional de
Saúde, confirmados pelo Comitê de Mortalidade Materna da região.

Na caracterização dos dados as variáveis analisadas foram: faixa etária, escolaridade, estado civil e raça.

\section{RESULTADOS}

Do total de 813 óbitos maternos no estado do Paraná (PR), no período avaliado, $2,58 \%$ (18) ocorreram no município de Maringá. O município de Maringá apresenta uma taxa média de morte materna de $61,64 / 100000$ NV que se aproxima ao do Estado 66,88/100000 NV.

Os óbitos por aborto no Estado do Paraná totalizaram no período, um percentual de $6,27 \%$ (51) dos óbitos maternos ocorridos (Gráfico 1). Observou-se uma ascensão gradativa no primeiro triênio com queda súbita até o ano de $2003(7,84 \%)$ e elevando-se novamente no ano consecutivo $(13,72 \%)$.

Gráfico 1: Distribuição dos casos de óbitos por aborto no Estado do Paraná, 1998 a 2004.

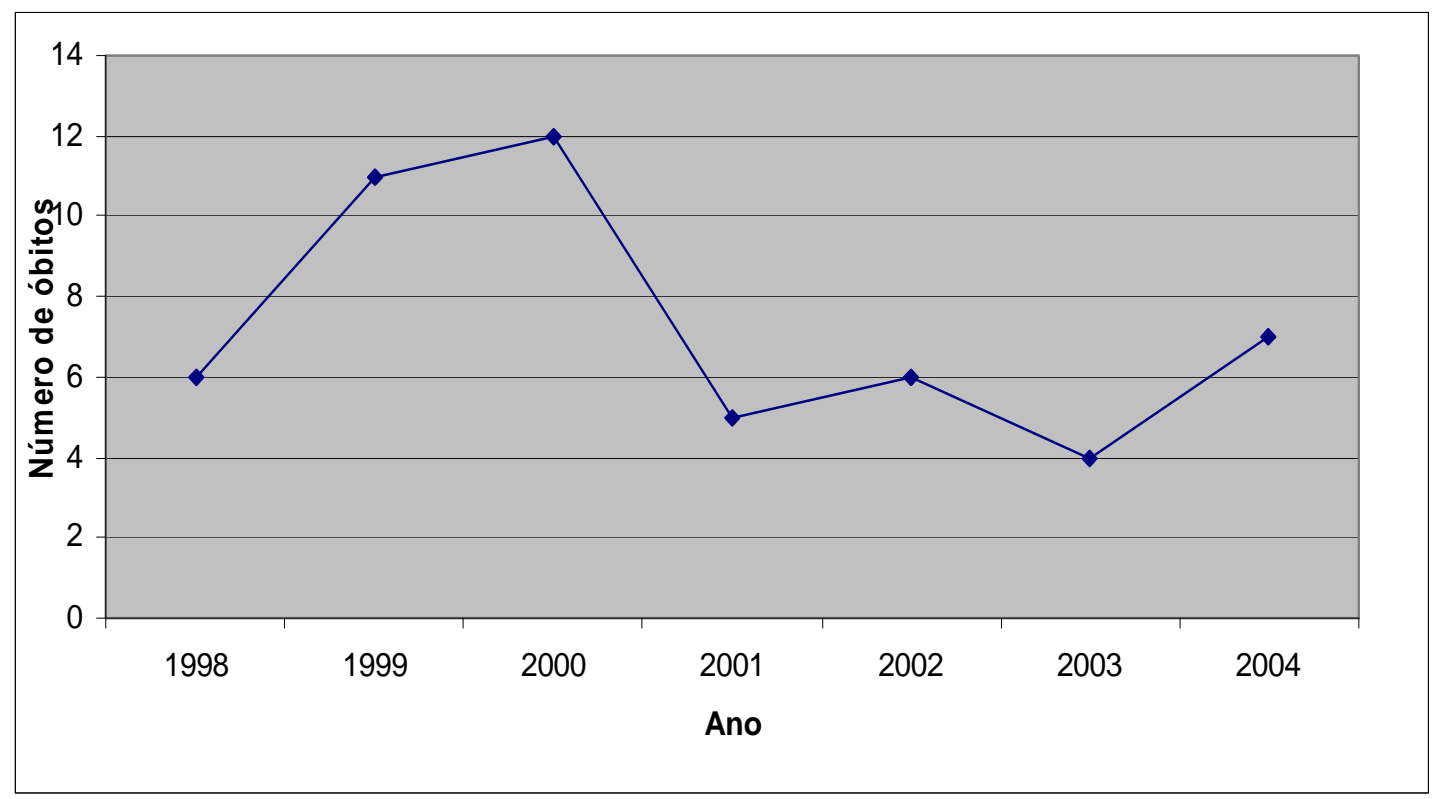

Fonte: SIM/MS/DATASUS 
Matos JC, Luz GS, Pelloso SM, Carvalho MDB. Mortalidade por aborto no Estado do Paraná: 1998 a 2004 . Revista Eletrônica de Enfermagem [serial on line] 2007 Set-Dez; 9(3):806-814. Available from: URL: http://www.fen.ufg. br/revista/v9/n3/v9n3a19.htm

Dentre os óbitos por aborto $47 \%$ (24) ocorreram em mulheres com idade entre 30 a 39 anos. Destaca-se que a adolescência (15 a
19 anos) é responsável por 23,5\% do total de óbitos (Tabela 1).

Tabela 1: Distribuição percentual dos óbitos por aborto segundo faixa etária no Paraná, 1998-2004.

\begin{tabular}{cccccccccc}
\hline Faixa Etária & $\mathbf{1 9 9 8}$ & $\mathbf{1 9 9 9}$ & $\mathbf{2 0 0 0}$ & $\mathbf{2 0 0 1}$ & $\mathbf{2 0 0 2}$ & $\mathbf{2 0 0 3}$ & $\mathbf{2 0 0 4}$ & $\mathbf{N}$ & Total \\
\hline $15-19$ & 1,96 & 1,96 & 7,84 & 5,88 & - & 1,96 & 3,92 & 12 & 23,52 \\
$20-24$ & 3,92 & 1,96 & 1,96 & - & - & - & 1,96 & 5 & 9,8 \\
$25-29$ & - & 5,88 & 3,92 & - & 1,96 & - & - & 6 & 11,76 \\
$30-34$ & 1,96 & 1,96 & 1,96 & - & 7,84 & 3,92 & 3,92 & 11 & 21,56 \\
$35-39$ & 3,92 & 7,84 & 5,88 & 3,92 & - & - & 3,92 & 13 & 25,48 \\
$40-44$ & - & 1,96 & 1,96 & - & 1,96 & 1,96 & - & 4 & 5,88 \\
\hline Total & 11,76 & 21,56 & 23,52 & 9,8 & 11,76 & 7,84 & 13,72 & 51 & 100 \\
\hline
\end{tabular}

Quanto à escolaridade, 31,36\% (16) possuíam de cinco a oito anos de estudo. Destaca-se que $27,52 \%$ (14) dos registros desta variável apresentaram-se como ignorados no SIM/MS (Tabela 2).

Tabela 2: Distribuição percentual dos óbitos por aborto segundo escolaridade no Paraná, 19982004.

\begin{tabular}{cccccccccc}
\hline Escolaridade & $\mathbf{1 9 9 8}$ & $\mathbf{1 9 9 9}$ & $\mathbf{2 0 0 0}$ & $\mathbf{2 0 0 1}$ & $\mathbf{2 0 0 2}$ & $\mathbf{2 0 0 3}$ & $\mathbf{2 0 0 4}$ & $\mathbf{N}$ & Total \\
\hline Analfabeto & 1,96 & 1,96 & - & 1,96 & - & - & - & 3 & 5,88 \\
1 a 4 anos & - & - & 3,92 & 1,96 & - & 1,96 & 3,92 & 6 & 11,76 \\
5 a 8 anos & - & 13,72 & 7,84 & - & 7,84 & - & 1,96 & 16 & 31,36 \\
9 a 11 anos & - & 1,96 & 5,88 & 5,88 & - & 3,92 & - & 9 & 17,64 \\
12 anos ou + & - & - & 1,96 & - & 1,96 & - & 1,96 & 3 & 5,88 \\
$\quad$ Ign & 9,8 & 3,92 & 3,92 & - & 1,96 & 1,96 & 5,88 & 14 & 27,52 \\
\hline Total & 11,76 & 21,56 & 23,52 & 9,8 & 11,76 & 7,84 & 13,72 & 51 & 100 \\
\hline
\end{tabular}

O estado civil foi bem distribuído entre solteiras e casadas, respectivamente, 45\% (23) e $41 \%$ (21). A raça branca predominou com $76,47 \%$ (39).

No município de Maringá, ocorreram 2 óbitos por aborto confirmados pelo Comitê de Mortalidade Materna da 15a RS.I sso representa $3,12 \%$ dos óbitos ocorridos no estado.
Os dois óbitos ocorridos no município de Maringá caracterizam-se por mulheres solteiras com mesmo nível de escolaridade (8-11 anos), porém com faixa etária distinta (18 e 42 anos) (Quadro 1). 
Matos JC, Luz GS, Pelloso SM, Carvalho MDB. Mortalidade por aborto no Estado do Paraná: 1998 a 2004 . Revista Eletrônica de Enfermagem [serial on line] 2007 Set-Dez; 9(3):806-814. Available from: URL: http://www.fen.ufg. br/revista/v9/n3/v9n3a19.htm

Quadro 1: Óbitos por aborto segundo características sociodemográficas, Maringá/PR, 1998-2004.

\begin{tabular}{|c|c|c|}
\hline Características & Mulher $\mathbf{X}$ & Mulher $\mathbf{Y}$ \\
\hline Causa & Aborto Infectado & Aborto Provocado \\
\hline Idade & 18 anos & 42 anos \\
\hline Estado Civil & Solteira & Solteira \\
\hline Escolaridade & 8 a 11 anos & 8 a 11 anos \\
\hline
\end{tabular}

Fonte: 15a RS/ Comitê de Mortalidade Materna

\section{SCUSSÃO}

No Estado do Paraná, os óbitos por aborto no período de 1998 a 2004, corresponderam a $6,27 \%$ (51) dos óbitos maternos. O Comitê Central de Mortalidade Materna $^{(4)}$ em um estudo no município de São Paulo verificou que a mortalidade por aborto apresentou um percentual maior em relação ao Estado do Paraná, com $8,85 \%$ mortes por aborto dentre todas as mortes maternas, no ano de 2000.

Constatou-se em uma pesquisa que no 1 응 semestre de 2002, o número de óbitos por aborto e a proporção na morte materna segundo região, foi a seguinte: Norte 3/11, $1 \%$; Nordeste 10/14,9\%; Sudeste $6 / 8,1 \%$; Sul $2 / 18,2 \%$; Centro Oeste $2 / 9,1 \%$. Na presente pesquisa, neste mesmo ano, ocorreram no Estado do PR 6/6, 25\% ${ }^{(8)}$. Os dados da região Sul a qual faz parte o PR, demonstrou que o elevado número de óbitos por aborto no Estado pode ter ocorrido em sua maioria no 2 응 semestre de 2002.

Estima-se que em 2005 o risco anual de abortos induzidos por 100 mulheres de 15 a 49 anos é de 2,73 na Região Nordeste e 1,28 na Região Sul. Além da diferença Nordeste/Sul, a Região Centro-Oeste, apresenta o maior risco: 2,81 abortos/100 mulheres ${ }^{(8)}$. As diferenças regionais caracterizam a efetividade dos programas de planejamento familiar na região Sul.

Em um levantamento das variações entre as datas de 1980 e 1995 dos coeficientes de aborto ilegalmente induzido e não especificado nas regiões do Brasil, a região Sul apresentou um valor decrescente de 0,8 óbitos por 100000 em 1995 nas mulheres de 10 a 54 anos. As regiões Norte e Nordeste também apresentaram a mesma taxa $(0,8)$, porém com maior incidência de subnotificações ${ }^{(13)}$.

Em países desenvolvidos, que fornecem serviços seguros para um aborto legal, o coeficiente de mortalidade por aborto não é maior que 1 óbito por 100000 abortos ${ }^{(14)}$. Este fato está longe da realidade brasileira à medida que a solução da mortalidade por aborto está muita além da legalização do abortamento, envolve aspectos educacionais, culturais e de cidadania dos profissionais da área da saúde e população.

As mortes maternas decorrentes de complicações por aborto ao longo do período estudado apresentaram variações significativas. Nos primeiros três anos $(1998,1999,2000)$ há uma elevação dos números absolutos. A partir daí eles caem até o ano 2003 quando apresentam nova elevação em 2004.

O aborto no Estado do Paraná revelou índices bem semelhantes entre as seguintes 
Matos JC, Luz GS, Pelloso SM, Carvalho MDB. Mortalidade por aborto no Estado do Paraná: 1998 a 2004 . Revista Eletrônica de Enfermagem [serial on line] 2007 Set-Dez; 9(3):806-814. Available from: URL: http://www.fen.ufg.br/revista/v9/n3/v9n3a19.htm

faixas etárias: 15-19 anos (23,52\%); 30-34 anos $(21,56 \%)$ e $35-39$ anos $(25,48 \%)$. Ainda que bem semelhantes, a faixa etária de 15-19 anos é a que se mostra significativamente mais preocupante à medida que é a fase de maior risco para provocar o aborto de modo inseguro e inadequado, contribuindo assim para as estatísticas do óbito materno no Brasil. Dados oficiais do Ministério da Saúde retratam um decréscimo na média de idade de mulheres que foram a óbitos por aborto, assim como a permanência de desigualdades regionais, com menor redução destas taxas nos estados nordestinos ${ }^{(10)}$.

A gravidez na adolescência, na maioria das vezes indesejada e sem apoio social e da família, contribui para o aumento do índice de aborto ilegal e óbito materno ${ }^{(15)}$.

A prática do aborto em adolescentes é maior que em mulheres adultas ${ }^{(16)}$. O aborto ocorre geralmente mais em adolescentes nulíparas do que em mulheres mais velhas que possuem um ou mais filhos ${ }^{(13)}$.

Os óbitos por aborto ilegal e não especificados constituem-se em maior número na idade entre 15-24 anos ${ }^{(13)}$. Pelo fato do início da atividade sexual estar se tornando cada vez mais precoce favorece a sua inserção nas estatísticas de algumas doenças (HPV, câncer cervical, DST's etc) e na mortalidade materna, principalmente por causas relacionadas ao aborto. A falta de informação e a utilização errônea de métodos contraceptivos contribuem para uma gravidez indesejada podendo levar a um aborto inseguro e inadequado em clínicas clandestinas ou por métodos caseiros. Em 1998, mais de 50 mil adolescentes foram atendidas no sistema público de saúde para curetagem pós-aborto, sendo cerca de 3 mil realizadas entre jovens com idade entre 10 e 14 anos ${ }^{(17)}$.

Estudo realizado com adolescentes que provocaram o aborto em um município na Bahia, constatou que $81,6 \%$ eram solteiras ${ }^{(18)}$. O presente estudo contraria esses dados, já que não houve diferença significativa nas mortes por abortos entre solteiras e casadas. Entretanto, as duas mortes que ocorreram no município de Maringá foram de mulheres solteiras.

Em relação à raça houve uma predominância da raça branca com 76,47 \% (39) dos óbitos maternos. Por se tratar de mortalidade por aborto, verifica-se que a questão da raça foi diferencial quando se discute a mortalidade materna. Há 50 anos, e ainda atualmente, o risco de mulheres negras e as de idade mais avançada morrerem por óbito materno é maior em relação às mulheres brancas ${ }^{(19)}$. Os dados do presente estudo não corroboram os achados de Martins.

Quanto à escolaridade os dados indicam que existe uma relação entre anos estudados e número de mortes maternas por complicações pós-aborto. Das mortes maternas por complicações pos aborto $49 \%$ eram de mulheres analfabetas ou que tinham entre 1 a 8 anos de estudo,17,64\% entre 9 e 11 anos de estudo e $5,88 \%, 12$ ou mais anos de estudo.

Os dois abortos ocorridos no município de Maringá, no período de estudo, foram classificados como infectado e provocado, segundo o Comitê de Mortalidade Materna da região. Esta característica permite determinálos como abortos intencionais.

As duas mulheres que morreram por complicações pós-aborto tinham idades 
Matos JC, Luz GS, Pelloso SM, Carvalho MDB. Mortalidade por aborto no Estado do Paraná: 1998 a 2004 . Revista Eletrônica de Enfermagem [serial on line] 2007 Set-Dez; 9(3):806-814. Available from: URL: http://www.fen.ufg. br/revista/v9/n3/v9n3a19.htm

dispares, ou seja, 18 e 42 anos e escolaridade entre 8 e 11 anos.

\section{CONCLUSÃO}

Dos 813 óbitos maternos no estado do Paraná no período de 1998 a 2004, 51 ocorreram por complicações pós-aborto e dois deles no município de Maringá.

Os resultados encontrados mostraram uma tendência de aumento do número de óbitos no primeiro triênio do período estudado, seguido de queda significativa e uma nova elevação.

No estado do Paraná a maior freqüência de morte por complicações do abortamento ocorreu mais entre mulheres em duas faixas etárias distintas: de 15 a $19(23,52 \%)$ e 30 a $39(47 \%)$ anos, com condição conjugal bem distribuída entre solteiras (45\%) e casadas (41\%). 49\% eram analfabetas ou tinham entre 1 a 8 anos de estudo.A raça branca predominou com 76,47\%.

No município de Maringá, os dois óbitos ocorridos caracterizam-se por mulheres solteiras com o nível de escolaridade (8-11 anos) semelhantes, porém com faixa etária distinta (18 e 42 anos).

A realidade no Estado do Paraná e município de Maringá/PR, constatado no presente estudo, enquadram-se nas estatísticas brasileira.

A evolução das mortes maternas por complicações do aborto no período estudado, aponta para a necessidade de uma ação urgente de caráter preventivo nos programas de saúde da mulher. Ações destinadas às adolescentes, orientando e colocando a disposição tecnologia que lhes permita vivenciar uma sexualidade saudável e sem riscos, evitando mortes prematuras. O trabalho de orientação e de educação a saúde precisa ser desenvolvido de tal modo, que favoreça o apoderamento do saber pela clientela para assim, tomar suas decisões de maneira consciente e voluntária. Este é o grande desafio da educação para a saúde que neste caso se torna maior, já que uma parcela considerável da população estudada tem baixa escolaridade, o que pode se tornar um complicador do processo.

O aborto, dentre todas as causas de morte, enfrenta dilemas culturais, religiosos, sociais, morais e éticos, entre outros. Esbarra em assuntos delicados e intocáveis que causa constrangimentos e controvérsias. Esses dilemas humanos fazem parte do cotidiano dos profissionais de saúde e contribuem decisivamente na assistência prestada à mulher, seja curativa, educativa ou preventiva.

A sua característica polêmica e controvertida contribui para que seja um dos maiores condicionantes na alta taxa de óbito materno e de internações, gerando altos custos para o sistema de saúde.

Ainda que o aborto seja atualmente um tema muito discutido e debatido em congressos, percebe-se a deficiência no avanço sobre a questão demonstrada claramente nos números de mortalidade materna por aborto.

\section{REFERÊNCI AS}

1. Pelloso SM, Tavares MSG. Evolução da mortalidade Materna na 15a Regional de Saúde de Maringá, Estado do Paraná, entre 1989 a 2000. Rev. Acta Scientiarum 2002; 24:783789.

2. Gomes FA, Nakano MAS, Almeida AM, Matuo YK. Mortalidade Materna na Perspectiva do Familiar. Rev da Escola de Enfermagem da USP 2006; 40 (1):50-56. 
Matos JC, Luz GS, Pelloso SM, Carvalho MDB. Mortalidade por aborto no Estado do Paraná: 1998 a 2004 . Revista Eletrônica de Enfermagem [serial on line] 2007 Set-Dez; 9(3):806-814. Available from: URL: http://www.fen.ufg.br/revista/v9/n3/v9n3a19.htm

3. Organização Mundial da Saúde. Classificação Estatística Internacional de Doenças e Problemas Relacionados à Saúde; 10ạ Revisão. CBCD, São Paulo 1995.

4. Comitê Central de Mortalidade Materna. Relatório sobre o ano 2000 2a ${ }^{a}$ versão. Coordenação de desenvolvimento da gestão descentralizada (COGEst-SMS). Atualizada em agosto de 2002.

5. Ministério da Saúde (BR). Manual dos Comitês de Mortalidade Materna. Área Técnica de Saúde da Mulher. Secretaria de Políticas de Saúde. Brasília (DF): Ministério da Saúde; 2002.

6. Laurenti R, Ferreira CEC. Diferenciais de Mortalidade Materna segundo Nível Sócioeconômico no Município de São Paulo. São Paulo: Faculdade de Saúde Pública, Universidade de São Paulo. (mimeo) 1995.

7. Pazero LC, Vega CEP, Marcus PAF, Barbosa SA, Boyaciyan K. Estudo da mortalidade materna no municipio de São Paulo durante o ano de 1996. Rev. Bras. Ginecologia e Obstetrícia 1998; 20 (7):395-403.

8. Laurenti R, Jorge MHPM, Gotlieb SLD. A mortalidade materna nas capitais brasileiras: algumas características e estimativa de um fator de ajuste. Rev. Bras. Epidemiologia 2004; 7 (4): 449-460.

9. Parpineli MA, Faúndes A, Cecatti JG, Surita FGC, Pereira BG, Passini Júnior R, Amaral E. Subnotificação da Mortalidade Materna em Campinas: 1992 a 1994. Rev. Bras. Ginecologia e Obstetrícia 2000; 22 (1):27-32.

10. Ministério da Saúde (BR). Atenção Humanizada ao Abortamento: norma técnica. Secretaria de Atenção à Saúde. Departamento de Ações Programáticas estratégicas. Brasília(DF): Ministério da Saúde; 2005.

11. Mariutti M.G, BOEMER MR. A mulher em situação de abortamento - um enfoque vivencial. Rev da Escola de Enfermagem da Universidade de São Paulo 2003; 2(37):59-71.

12. IPAS. Mortalidade Materna [serial on line] 2003 [cited 2006 jun 12]; Available from: URL: http://www.ipas.org. br/mortalidade. html.

13. Lima BGC. Mortalidade por causas relacionadas ao aborto no Brasil: declínio e desigualdades espaciais. Rev Panam Salud Publica/Pan Am J Public Health 2000; 7(3): 168-172.

14. The Alan Guttmachher Institute. Sharing responsability: women, society and abortion: wordwide. [S.I.]: Special report 1999, $57 \mathrm{p}$.

15. Ponte Júnior GM, Ximenes Neto FRG. Gravidez na adolescência no município de Santana do Acaraú - Ceará - Brasil: uma análise das causas e riscos. Rev. Eletrônica de Enfermagem [serial on line] 2004 [cited 2006 aug 02]; 6 (1):25-37. Available from: URL: http://www.fen.ufg. br/ revista/revista6 1/f3 gr avidez.html.

16. Gontijo DT, Medeiros M. Gravidez / maternidade e adolescentes em situação de risco social e pessoal: algumas considerações. Revista Eletrônica de Enfermagem [serial on line] 2004 [cited 2006 aug 02]; 6 (3). Available from: URL: http://www.fen.ufg. br/revista/revista6_3/12_R evisao2.html.

17. Ministério da Saúde (BR). A adolescente grávida e os serviços de saúde do município. Brasília (DF): Ministério da Saúde; 1999.

18. Souza VLC, Correa MSM, Souza SL, Beserra MA. O ABORTO ENTRE ADOLESCENTES. Rev. Latino-Am. Enfermagem 2001; 9 (2): 42-47.

19. Martins AL. Diferenciais raciais nos perfis e indicadores de mortalidade materna para o Brasil. Trabalho apresentado no XIV Encontro Nacional de Estudos Populacionais, ABEP: 2004 sep 20-24; Caxambu (MG), Brasil.

Artigo recebido em 23.05.07

Aprovado para publicação em 10.12.07 